\title{
Smoothing and Reconstruction Strategy for Part Repair Using a Laser Displacement Sensor
}

\author{
Zhenhui Shen ${ }^{1, *}$, Shuanqiang Yang ${ }^{1}$, Jianxiong Chen ${ }^{2}$ and Jun $\mathrm{Ma}^{3}$ \\ ${ }^{I}$ Engineering College, Fujian Jiangxia University, Fuzhou 350108, China \\ ${ }^{2}$ School of Mechanical Engineering and Automation, Fuzhou University, Fuzhou 350108, China \\ ${ }^{3}$ Department of Mechanical and Manufacturing Engineering, University of Calgary, Calgary T2N 1N4, Canada
}

Received 3 August 2018; Accepted 31 October 2018

\begin{abstract}
The reconstruction of the profile curve of damaged parts in the absence of an original technical drawing is an important process of part repair. Part damages are complicated and changeable. To reduce the material removal rate during repair and maintain a smooth and flawless profile curve after being repaired, a smoothing and reconstruction strategy using a laser displacement sensor to measure the profile curve was proposed in this study. This strategy established an adaptive measurement mechanism by building an automatic measurement motion platform. A staged repair and smoothing strategy of the profile curve measurement data was constructed on the basis of the locally weighted scatterplot smooth. A calculation model of a part repair processing curve was built by using the Akima interpolation. Moreover, a case study was conducted to verify the reconstruction accuracy of the strategy and smoothness of the reconstruction curve. Results demonstrate that the automatic measurement motion platform and adaptive measurement mechanism can realize an adaptive measurement of different profile structural shapes. The staged repair and smoothing strategy can repair the measurement error of profile curve measurement data, profile defects, and other abnormal data while maintaining the smoothness of the profile curve. After repair, the variation range in the measurement data error at different points of the profile curve decrease from $(-1.4913,+0.0351)$ to $(-0.3511,+0.3715)$. Akima interpolation can not only increase the maximum error of the processing curve but also decrease the material removal rate effectively and protect the smoothness of the profile curve. This study provides important guidance to increase the part repair efficiency and accuracy and realize the automatization of part repair.
\end{abstract}

Keywords: Curve measurement, Smoothing, Laser displacement sensor, Locally weighted scatterplot smooth, Akima interpolation

\section{Introduction}

Parts that work in tough environment frequently develop serious abrasion, deformation, and even fracture. This phenomenon is disadvantageous to users and typically brings high economic losses. Repair of damaged parts can not only bring remarkable benefits to enterprises but also effectively prolong the service life of these parts. However, the damaged parts have uncontrollable quality factors and highly complicated shape, size, and structure. As an automation technology, reverse engineering is extensively used in the new part design, existing part reproduction, damaged part repair, digital model verification, and accuracy improvement This technology has been extensively believed as an important topic in a product design process [1-4]. When introducing reverse engineering into the repair of damaged parts, an original technical drawing is unnecessary and claims a significantly low time consumed and expenditures, especially to a complicated part model.

In contrast to the traditional design order, reverse engineering starts by acquiring model data through existing part measurement. On this basis, the reconstruction part model exploits $\mathrm{CAD} / \mathrm{CAM} / \mathrm{CAE}$ technologies. Existing measurement methods of parts can be divided into contact

*E-mail address: shenzhenhui@fijixu.edu.cn

ISSN: 1791-2377 @ 2018 Eastern Macedonia and Thrace Institute of Technology. All rights reserved. doi:10.25103/jestr.115.19 and noncontact. The noncontact measurement method is extensively used in the mechanical industry given the protection of an objective surface, high measurement accuracy, and large measurement range $[5,6]$. Noncontact measurement in reverse engineering mainly focuses on $2 \mathrm{D}$ image processing $[7,8]$ and $3 \mathrm{D}$ scanning data processing [911]. The 2D image measurement method causes missing nominal data considering the inconsistency in reflection light, shooting angle, and focal length, thereby influencing followup data analysis. Thus, this method has high requirements for image acquisition devices. Although the 3D scanning measurement method can acquire detailed features effectively, this method still requires a large data file to store dense point cloud data, which proposes remarkable challenges to computing efficiency and claims high hardware cost. Measurement data quality directly influences the repair quality of parts. In comparison with 2D image and 3D scanning measurements, measurement method based on a single-point laser displacement sensor is widely used in industrial detection given the high measurement accuracy, small data size, favorable frequency response, and insensitivity to environmental light source [12-14]. The use of the single-point laser displacement sensor for part repair and realization of profile curve measurement and repair and smoothing of measurement data are problems that must be solved urgently. Given that various damaged parts have different profiles, an adaptive trajectory planning of the laser displacement sensor must be established to maintain the 
target measuring point in the effective measurement range of the laser displacement sensor constantly. Considering laser reflection at the smooth surface, analog-to-digital conversion (ADC), and surface defects of parts, abnormal data in the measurement data are typically observed and must be repaired, smoothed, and reconstructed, thus upholding the consistency of the measurement data with the original curve. Moreover, repair, smoothing, and reconstruction methods of the profile curve must have low computation cost, favorable robustness, and high reliability for online running and adaption to complicated environment.

On this basis, the automatic measurement motion platform and adaptive measurement mechanism based on the laser displacement sensor are constructed. The staged repair and smoothing strategy of profile curve measurement data are investigated on the basis of locally weighted scatterplot smooth (LOWESS). A calculation model of the part repair processing curve is constructed by using Akima interpolation to provide a smoothing and reconstruction method based on the profile curve measured utilizing the laser displacement sensor. Study conclusions offer several references to increase part repair efficiency and accuracy and improve the automatization level of part repair.

\section{State of the art}

Considerable studies on profile reconstruction for part repair have been reported. Zheng et al. suggested to acquire wearing positions by comparing point cloud data and nominal model on the basis of reverse engineering technology [15]. These researchers also realized an automatic turbine blade and blisk repair strategy through laser welding and cladding. However, this technology depended on the nominal model and did not study profile smoothness after repair. Gao et al. proposed an integrated adaptive repair method on the basis of reverse engineering for component repair of the aerospace industry [16]. The reconstruction of a solid model did not depend on a nominal model. However, the reconstruction neglected profile smoothness after repair. Moreover, this reconstruction had certain disadvantageous, including time-consuming and poor online performance. Bagci mentioned the advantages of reverse engineering technologies in part repair and reproduction through three application cases [17]. Reverse engineering could reconstruct the continuous surface model of $\mathrm{C} 1$ without the original CAD model. However, automatizing the repair process was not studied, and the applied surface reconstruction algorithm reported low repair efficiency. Yilmaz et al. presented a repair strategy for complicated geometric parts and expensive components on the basis of the reverse engineering technology [18]. This strategy integrated noncontact digital measurement, adaptive free-form surface reconstruction, and multiaxis milling and realized the adaptive measurement of the data model. However, the applied surface modeling method presented a low automation degree and was unsuitable for online repair. On the basis of structural restoration method, Shen et al. constructed a high-quality 3D model using low-quality data from a consumption-level scanning device [19]. This model has achieved a solid modeling of the assembly from local to global and from upper to bottom of a defined part. However, this reconstruction method was inapplicable to parts with unknown structures. Jones et al. proposed the flexible precise reproduction system for repair of damaged parts that integrate laser cladding, mechanical processing, and online scanning [20]. However, these researchers used a contact measurement with poor efficiency and automatization level. $\mathrm{Wu}$ et al. introduced geometric reconstruction methods and repair algorithms for repairing turbine components [21]. These researchers emphasized that data sharing in a highly efficient and accurate geometric reconstruction algorithm and the repair process are two important research directions of turbine component repair in the future. Wilson et al. acquired the parameterized repair quantity through the Boolean operation between the original defect and the reconstruction models and realized the turbine airfoil defect repair on the basis of a semiautomatic geometric reconstruction algorithm and laser rapid prototyping [22]. However, this repair method had several disadvantages, such as large calculation load, low automation degree, and low repair efficiency. Zhuo et al. suggested to calculate the damaged part repair model through the difference chart between the reconstruction and the nominal models, thereby determining the repairing robot processing path [23]. However, this method depended on the nominal model, had low repair efficiency, and easily caused poor repair accuracy given inconsistency between the reconstruction and nominal model coordination systems. Huang et al. proposed a method for crude extraction of damage boundaries based on curvature features, fine extraction of damaged boundaries based on vertex normal vector features, and key characteristic size extraction of damage boundaries [24]. Moreover, these researchers reproduced damaged parts through laser cladding. However, the operation was relatively complicated and showed low automatization level. $\mathrm{Li}$ et al. put forward the repair strategy that integrates 3D surface data collection, nominal model reconstruction, fine registration, extraction of additive/subtractive repair, tool path generation, and actual machining process to increase reliability and efficiency of the manual repair process [25]. However, this strategy was challenged by big 3D surface data, complicated calculations, and low automation level. Sokół et al. repaired damaged parts in the assembly process through digital reverse modeling and 3D printing technologies [26]. However, these researchers did not analyze the smoothness of the model surface. The repair process claimed high labor operation intensity, thereby indicating low repair efficiency. Zhang et al. proposed an automatic damaged surface modeling method [27], which extracted missing volume through a ray casting method, generated tool path, and repaired the missing volume through laser metal deposition forming. This method depended on the nominal model. The repair algorithm was relatively complicated and achieved low repair efficiency. Zheng et al. repaired the damaged parts by using a coordinate measuring machine, defect model reconstruction, and decision-making for process selection for the repair [28]. However, the adaptive measurement remains unexplored, and the measurement efficiency was low. The conclusion drawn from this research had not been applied to practical engineering.

These research findings are mainly related to surface point cloud acquisition in part repair, 3D solid model reconstruction, repair technology selection, and tool path generation. However, adaptive profile data acquisition of laser displacement sensor and smoothing and reconstructing the profile curve, especially surface repair of smooth rotational parts, have been rarely studied. In the present study, the measurement motion platform and adaptive measurement mechanism are constructed using laser displacement sensor, motion control card (MCC), axis of 
movement, and industrial personal computer (IPC). The staged repair and smoothing strategy of the profile curve measurement data are established by the LOWESS. Finally, the calculation model of the part repair processing curve is constructed through Akima interpolation. The results provide references for an automatic smooth rotary surface repair.

The remainder of this study is organized as follows. Section 3 describes the measurement motion platform and adaptive measurement mechanism. The staged repair and smoothing strategy of the profile curve measurement data based on the LOWESS and the calculation model of the part repair processing curve based on Akima interpolation are constructed. Section 4 analyzes the constructed adaptive measurement mechanism, staged repair and smoothing strategy, and calculation model of the part repair processing curve based on the hub end surface repair. The feasibility and validity of the smoothing and reconstruction strategy on the basis of the measurement profile curve of the laser displacement sensor in a part repair are verified. Section 5 summarizes the conclusions.

\section{Methodology}

\subsection{Profile curve measurement}

The smooth rotational part surface repair is generally realized by turning along the profile curve. The motion platform structure of profile curve measurement is illustrated in Fig. 1. This structure is composed of integrating the IPC, MCC that contains the ADC module, laser displacement sensor, and axis of motion.

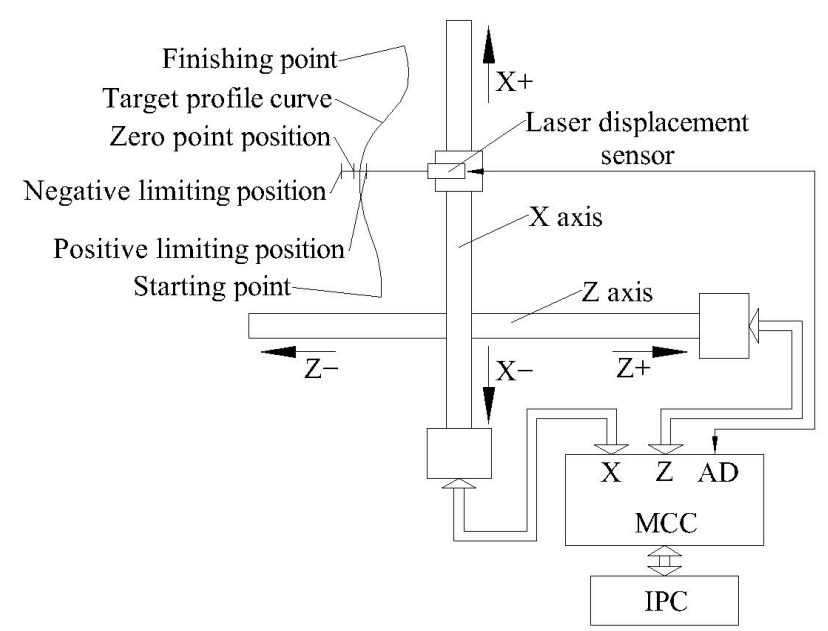

Fig. 1. Measurement motion platform structure

The IPC sends instruction sequences to MCC and reads the current coordinate and ADC values from the MMC. During the measurement process, the laser displacement sensor measures from the starting point $P_{\mathrm{s}}\left(x_{\mathrm{s}}, z_{\mathrm{s}}\right)$ to the finishing point $P_{\mathrm{f}}\left(x_{\mathrm{f}}, z_{\mathrm{f}}\right)$ along the $\mathrm{X}$ and $\mathrm{Z}$ axes driven by the MMC. Measurement data at different points $P_{k}\left(x_{k}, z_{k}\right)$ $(k=1,2, \mathrm{~L} \mathrm{~K})$ on the profile curve can be calculated using Eq. (1):

$\left\{\begin{array}{l}x_{k}=x_{\mathrm{c}} \\ z_{k}=z_{\mathrm{c}}+m_{\mathrm{c}}\end{array}\right.$ where $P_{c}\left(x_{c}, z_{c}\right)$ is the current coordinate value of the $\mathrm{X}$ and $\mathrm{Z}$ axes. $m_{c}$ is the current ADC value, which represents the current measurement value of the laser displacement sensor. Therefore, $P_{1}$ is the measured value of the profile curve at the starting point $P_{\mathrm{s}}$, whereas $P_{\mathrm{K}}$ is the measured value of the profile curve at the finishing point $P_{\mathrm{f}}$. To protect the validity of the measured value of the laser displacement sensor, target measuring points on the profile curve must be made into the effective measurement range determined by the negative and positive limit positions of the laser displacement sensor in each measurement. Thus, the adaptive point-by-point measurement mechanism of the profile curve is constructed (Fig. 2). With each movement to the next measuring point, the Z-coordinate can be shifted in accordance with the measured value of the current laser displacement sensor, thus realizing the adaptive measurement of the profile curve. The adaptive point-bypoint measurement steps of the profile curve are introduced as follows:

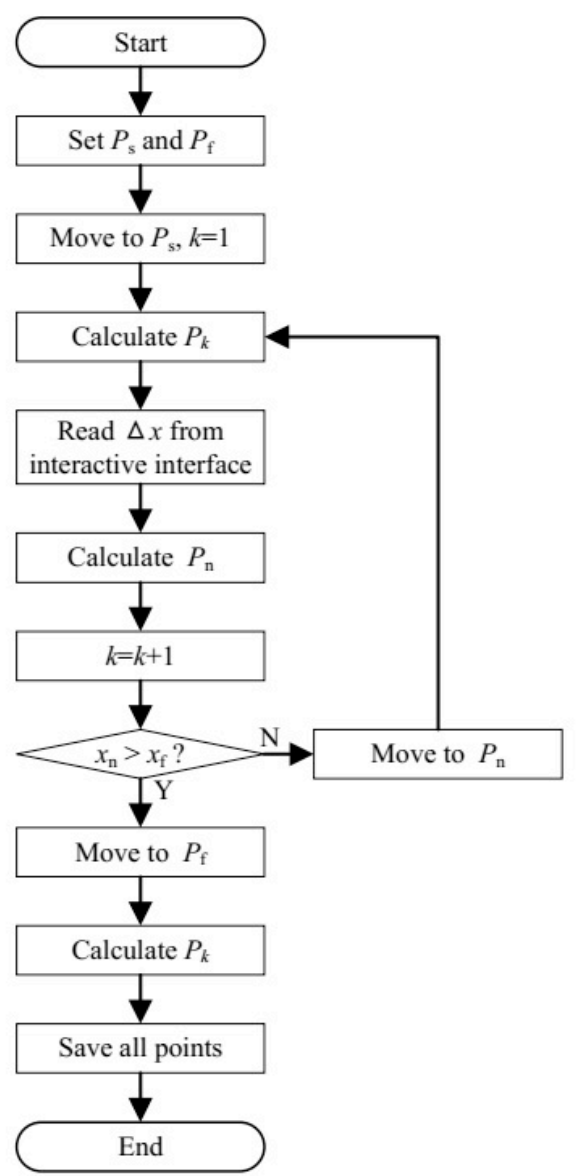

Fig. 2. Adaptive point-by-point measurement mechanism

Step 1) The coordinate values of $P_{\mathrm{s}}$ and $P_{\mathrm{f}}$ are set, and the axis of motion is moved to $P_{\mathrm{s}}$. Meanwhile, $k$ is set to 1 .

Step 2) $P_{k}$ is calculated, and the measured step length $\Delta x$ from the interaction interface is read, thus calculating the coordinate position $P_{\mathrm{n}}\left(x_{\mathrm{n}}, z_{\mathrm{n}}\right)$ of the next measuring points using Eq. (2):

$\left\{\begin{array}{l}x_{\mathrm{n}}=x_{k}+\Delta x \\ z_{\mathrm{n}}=z_{k}\end{array}\right.$ 
Then, $k$ has achieved an auto-increment.

Step 3) $x_{\mathrm{n}}$ in $P_{\mathrm{n}}$ is judged if higher than $x_{\mathrm{f}}$ in $P_{\mathrm{f}}$. If yes, then the coordinate axis is moved to $P_{\mathrm{f}}$, and the measured value $P_{k}$ (equivalent to $P_{\mathrm{K}}$ ) is calculated. At this point, the overall curve of the damaged parts has finished the adaptive measurement. Otherwise, go to Step 2).

\subsection{Measurement data repair and smoothing}

Given that parts have defect regions and the reflection by the smooth part surface and ADC can easily cause measurement errors, measured data of a profile curve are frequently mixed with abnormal data. Thus, considerable errors of local changes between the measurement and the nominal curves are present. These errors not only lead to high material removal rate and low structural strength at a repair but also cause uneven and unacceptable part surface after the repair. Therefore, the repair and smoothing of abnormal data are necessary. Given that the repair must be used in an online complicated environment, the repair and smoothing algorithm of abnormal data must have the advantages of low calculation cost, favorable robustness, and high reliability. Therefore, the staged repair and smoothing strategy depicted in Fig. 3 is used to increase the consistency between the profile and nominal curves and maintain the part smoothness.

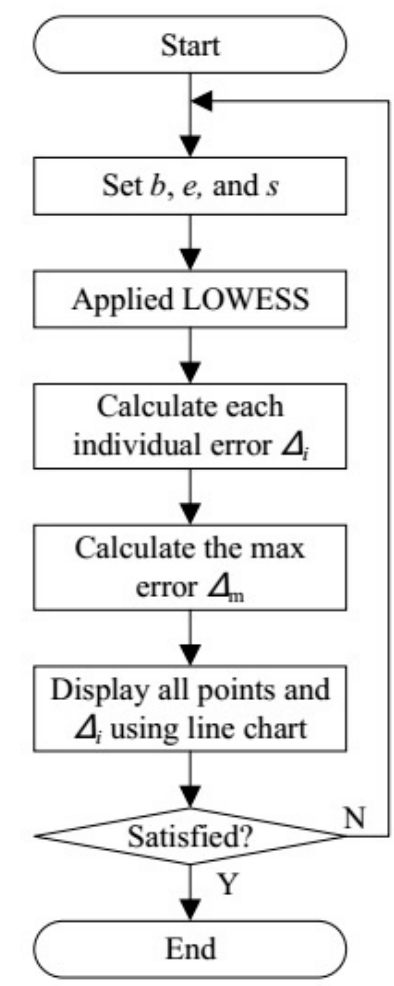

Fig. 3. Staged repair and smoothing strategy

The staged repair and smoothing strategy are realized on the basis of the LOWESS. The LOWESS can not only repair measurement data containing measurement error and surface defects but also be used in measured data smoothing [29-30]. The staged repair and smoothing strategy realize the measured data repair and smoothing of the profile curve on the basis of the repair and smoothing of local data segments. The local data segments are determined by the initial index value $b$ and the final index value $e$, where $1 \leq b<e \leq \mathrm{K}$. The LOWESS repairs and smoothes $z_{i}$ of different $P_{i}$ in the local data segments through the locally weighted linear regression, where $b \leq i \leq e . z_{i}$ at different points after repair and smoothing is determined by the Z-coordinate $z_{j}$ of the adjacent points in the span $s$. In the LOWESS, the initial weight $\omega_{j}$ of the adjacent points is calculated by the tricube function in Eq. (3):

$\omega_{j}=\left(1-\left|\frac{z_{i}-z_{j}}{d_{\mathrm{m}}}\right|\right)$

where $d_{\mathrm{m}}$ is the maximum distance between $z_{i}$ in the span $s$ along the Z-direction and the adjacent point $z_{j}$. On this basis, regression estimation can be implemented using $\omega_{j}$, and the residual error $r_{i}$ is calculated. The calculation formula of a robust estimated weight $\delta_{i}$ in Eq. (4) is gained.

$$
\delta_{i}=\left(1-\frac{r_{i}}{6 \operatorname{Median}\left(\left|r_{1}\right|,\left|r_{2}\right|, \mathrm{K},\left|r_{n}\right|\right)}\right)
$$

where $n$ is the number of measured data points in the span $s$, and median is the median filtering. Subsequently, the absolute value $\Delta_{i}$ of the error of $z_{i}$ at different points before and after the LOWESS is calculated. Then, the maximum $\Delta_{\mathrm{m}}$ among $\Delta_{i}$ is calculated. Furthermore, all repair and smoothing points and $\Delta_{i}$ of all points are presented in the linear diagram. The repair and smoothing results are judged as either satisfying or not. If yes, then end the repair and smoothing. Otherwise, $b, e$, and $s$ are reset for additional stages of repair and smoothing by using the LOWESS. Through the staged repair and smoothing, the measurement error of the Z-coordinate of the profile curve and defect data is repaired and smoothed. Moreover, the maximum error between the profile and the nominal curves decreases dramatically.

\subsection{Reconstruction of the profile curve}

The measured data of the profile curve have been repaired and smoothed by the staged repair and smoothing strategy. However, the measurement step length is unsuitable to be used as the processing step length directly. Thus, an interpolation of the repair and smoothing curve is required to reconstruct the profile curve that is appropriate for the processing step length. The reconstructed profile curve must be consistent with the repair and smoothed data, and no local concaves or convexes are permitted. Therefore, the Akima interpolation is used for reconstructing the profile curve. Akima interpolation constructs the segmented cubic polynomial between every two adjacent interpolation points $P_{i}$ and $P_{i+1}$. Moreover, it passes through every interpolation point $P_{i}$ strictly, thus preventing unnatural wiggles [31-32]. The use of Akima interpolation on the repair and smoothing data can reconstruct a smooth and flawless profile curve. In Akima interpolation, the coefficients in the polynomial are determined in accordance with the coordinate value and slope of relevant $P_{i}$ and $P_{i+1}$. The slope $t_{i}$ of Point $P_{i}$ is determined locally by using five adjacent points with $P_{i}$ in the center and can be calculated using Eq. (5): 
$t_{i}=\frac{\left|u_{i+1}-u_{i}\right| u_{i-1}+\left|u_{i-1}-u_{i-2}\right| u_{i}}{\left|u_{i+1}-u_{i}\right|-\left|u_{i-1}-u_{i-2}\right|}$

where $u_{i}$ is the slope of the segment $\overline{P_{i} P_{i+1}}$ and can be expressed as follows:

$u_{i}=\frac{z_{i+1}-z_{i}}{x_{i+1}-x_{i}}$

When calculating the starting and finishing points of the profile curve, several hypotheses are established to $u_{0}, u_{-1}$,

$u_{\mathrm{K}}$, and $u_{\mathrm{K}+1}$.

$u_{0}=2 u_{1}-u_{2}$

$u_{-1}=2 u_{0}-u_{1}$

$u_{\mathrm{K}}=2 u_{\mathrm{K}-1}-u_{\mathrm{K}-2}$

$u_{\mathrm{K}+1}=2 u_{\mathrm{K}}-u_{\mathrm{K}-1}$

In conclusion, during Akima interpolation, a cubic polynomial in each segment between $P_{i}$ and $P_{i+1}$ can be expressed as follows:

$z=A_{i}+B_{i}\left(x-x_{i}\right)+C_{i}\left(x-x_{i}\right)^{2}+D_{i}\left(x-x_{i}\right)^{3}$

where

$A_{i}=z_{i}$

$B_{i}=t_{i}$

$C_{i}=\frac{\frac{3\left(z_{i+1}-z_{i}\right)}{x_{i+1}-x_{i}}-2 t_{i}-t_{i+1}}{x_{i+1}-x_{i}}$

$D_{i}=\frac{\frac{-2\left(z_{i+1}-z_{i}\right)}{x_{i+1}-x_{i}}+t_{i}+t_{i+1}}{\left(x_{i+1}-x_{i}\right)^{2}}$

Thus far, the complete smooth profile curve is reconstructed to the part repair. Each interpolation points on the curve are only influenced by locally adjacent six points. When the reconstructed profile curve is adopted for processing, the renewal parts can be gained.

\section{Result Analysis and Discussion}

The feasibility and validity of the proposed strategy are verified by the hub end surface repair. Automobiles have become an important transportation tool. With the rapid development of the automobile industry, the aluminum alloy wheel hub has become safe, comfortable, convenient, and flawless; furthermore, it is highly appreciated by numerous consumers [33]. However, a hub will surely be scratched, worn, or damaged after being used extensively. Therefore, hub repair is an urgent demand. Hub repair frequently lacks an original technical drawing. Under this circumstance, the traditional hub repair mainly uses a numerically controlled (NC) lathe for processing and constructs a processing curve manually on the basis of operation experiences with low operational efficiency and strong human influences. Consequently, a large error between the processing and the original curves is present, thereby resulting in a large material removal rate and poor smoothness and appearance of an end surface after repair.

On the basis of the measurement motion platform structure proposed in Section 3.1, the measurement motion platform of the hub end surface profile is constructed by retrofitting the NC lathe (Fig. 4). The hub is installed on the platform shaft and laser displacement sensor on the tool carrier of the platform; this set up is convenient for assembly and disassembly. During repair processing, a hub end surface turning can be performed by replacing the laser displacement sensor with a turning tool, thus indicating a high working efficiency. Subsequently, the hub repair of a presupposed end surface profile curve is used as the study case. After damaging the hub end surface, its profile curve is measured through the adaptive point-by-point measurement mechanism discussed in Section 3.1. During the measurement, the measurement step length is set at a constant of $0.25 \mathrm{~mm}$, and the $\mathrm{K}$ value reaches 3301 after the measurement. The original curve, measurement curve of the hub, and their errors are demonstrated in Fig. 5. The measured profile curve contains flat and steep sections. However, it can accomplish a measurement adaptively on the measurement motion platform, without any artificial intervention. The Z-coordinate error at different measuring points changes between -1.4913 and $+0.0351 \mathrm{~mm}$. The measurement error is different at various positions. In particular, given that a damaged region on the hub is present, the Z-coordinate error is significantly higher in $P_{i}$ than in the rest of the positions when $x_{i}$ is between $(75,85)$ and $(135,155)$. Furthermore, if the only error caused by a laser reflection is considered, then a large error at points with the Z-coordinate changes more violently than the adjacent points, such as when $x_{i}$ is between 30 and 45 . The unevenly distributed Z-coordinate error and defects will cause poor hub appearances, such as convexes or concaves, after processing. Moreover, it will increase the material removal rate significantly. The measurement data cannot be used directly in generating the processing curve. Thus, the repair and smoothing of the measurement data mixed with abnormal data are required.

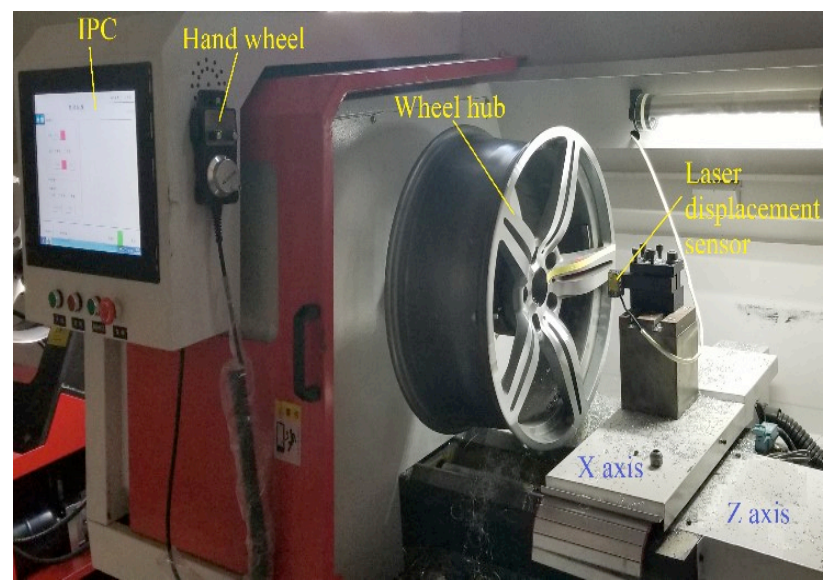

Fig. 4. Movement motion platform of a hub end surface profile 


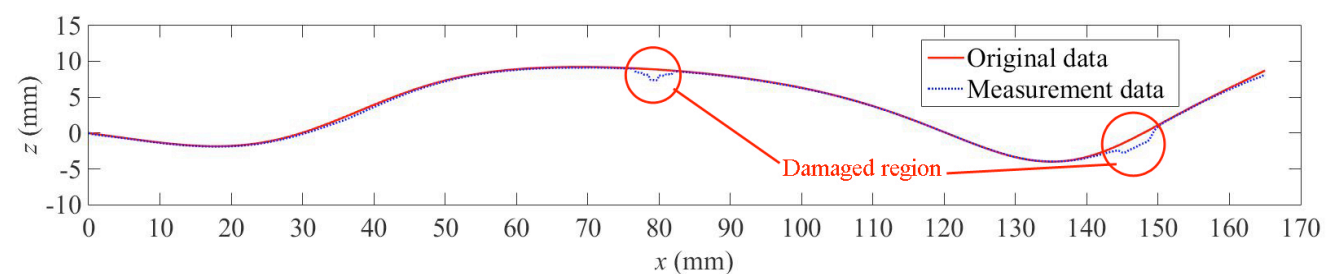

(a)

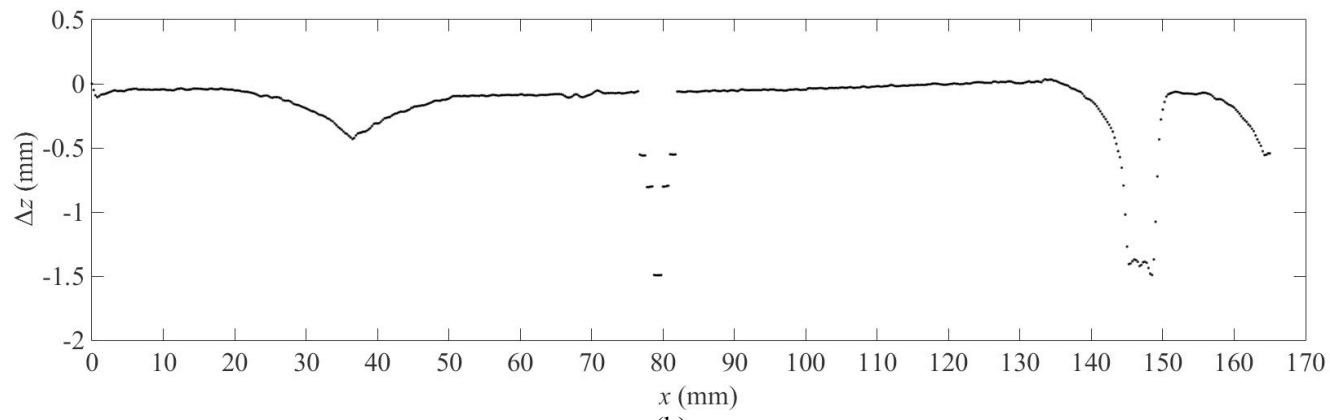

(b)

Fig. 5. Contrastive analysis between the measurement and the original curves

(a) Measurement and original curves (b) Errors between the measurement and the original curves

In accordance with the staged repair and smoothing strategy presented in Section 3.2 and the process summarized in Table 1, the staged repair and smoothing of the hub end surface profile measurement data are implemented using the LOWESS. In each repair stage, only $b, e$, and $s$ need to be set to different values and execute the LOWESS until the requirement is satisfied. The operation is simple, and the calculation efficiency is high. The repair process covers nine stages. The first six stages are mainly used to repair and smooth defects and errors on the profile curve, whereas the other three stages mainly realize the repair and smoothing of the whole curve. The contrast analysis results between the measurement and the original curves are exhibited in Fig. 6. The measurement curve remains consistent with the original curve, thereby indicating that the defects at the flat and steep regions are repaired, without distorting the global convex and concave characteristics of the curve. The maximum error of the Zcoordinate is decreased significantly. Errors at different measurement points change between -0.3511 and +0.3715 $\mathrm{mm}$, thus indicating the low material removal rate in the repair. In conclusion, the staged repair and smoothing strategy can realize the repair and smoothing of different types of profile curves, including flat, steep, concave, convex, and combined. This strategy is characterized by high repair quality, wide application range, and high reliability.

Table. 1. Stages of the repaired and smoothed measurement data

\begin{tabular}{c|c|c|c|c}
\hline Stage & B & e & s & Apply Times \\
\hline 1 & 20 & 36 & 0.8 & 5 \\
2 & 20 & 36 & 0.1 & 4 \\
3 & 80 & 200 & 0.2 & 2 \\
4 & 280 & 360 & 0.8 & 2 \\
5 & 240 & 400 & 0.8 & 1 \\
6 & 560 & 660 & 0.3 & 9 \\
7 & 0 & 3301 & 0.015 & 1 \\
8 & 0 & 3301 & 0.0015 & 3 \\
9 & 0 & 3301 & 0.017 & 1 \\
\hline
\end{tabular}

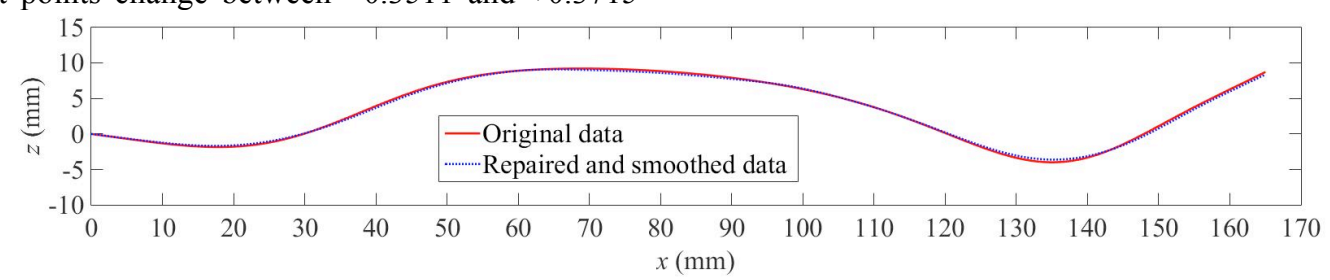

(a)

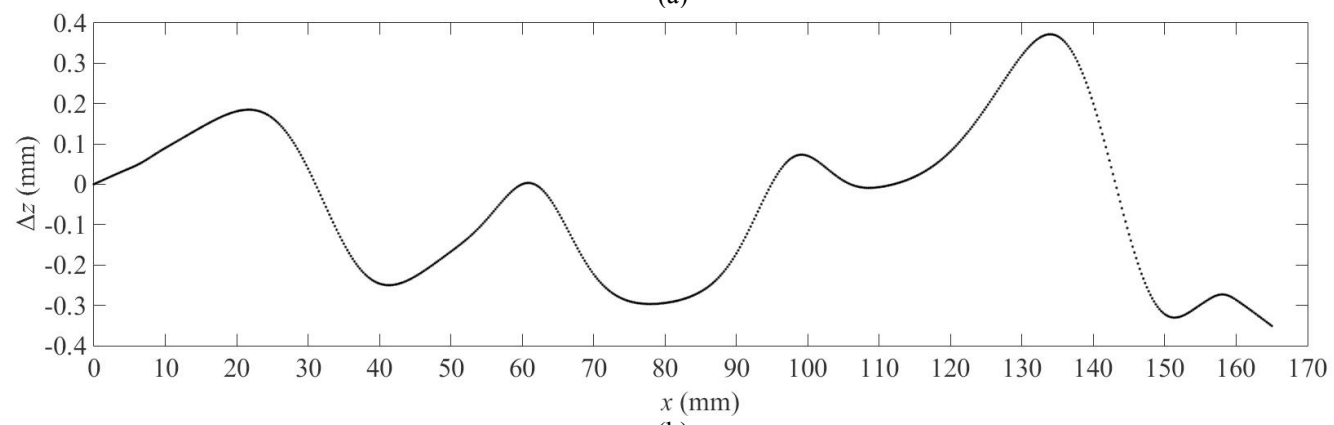

(b)

Fig. 6. Comparison between repaired and smoothed and original curves (a) Repaired and smoothed and original curves (b) Error between the repaired and smoothed and original curves 
Given that the measurement step length is too long to be used directly for processing, the Akima interpolation discussed in Section 3.3 is used to reconstruct the profile curve. The interpolation step length is set to $0.01 \mathrm{~mm}$. The comparison between the reconstruction and the original profile curves is displayed in Fig. 7. Given that the Akima interpolation passes through each interpolation point strictly, the reconstruction profile curve is consistent with the repaired and smoothed profile curve. The Z-coordinate error curves are also consistent, and the error ranges from -0.3511 $\mathrm{mm}$ to $+0.3715 \mathrm{~mm}$. The maximum error is not increased. Evidently, the reconstruction profile curve based on the
Akima interpolation is characterized by high reconstruction accuracy, minimal calculation cost, and high reliability. In Fig. 8, the first-order numerical differentiation curve changes continuously in full length, thereby indicating that the reconstruction curve is smooth. The hub end surface repair based on the reconstructed curve can contribute a flawless and smooth surface, consistent with the original curve. Therefore, this reconstruction method will not increase the maximum error of the Z-coordinate of the curve and protects the smoothness of the reconstruction curve.

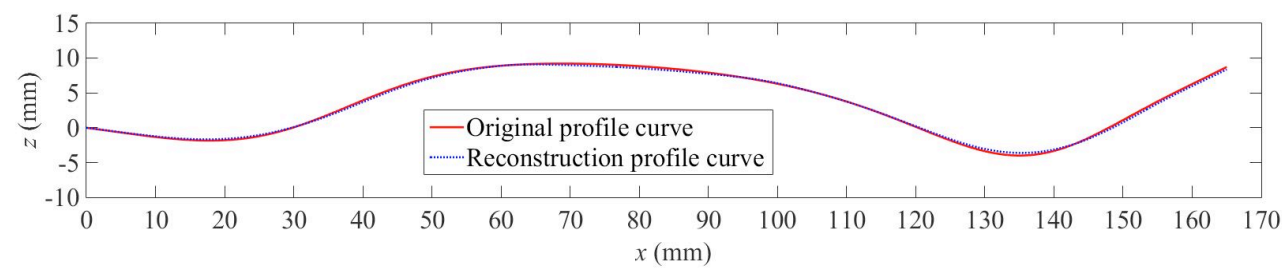

(a)

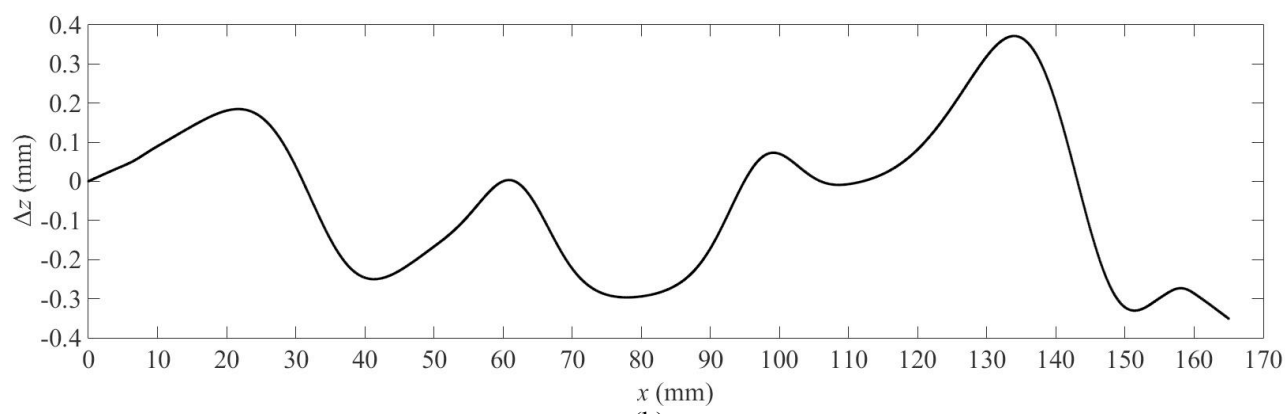

(b)

Fig. 7. Comparison between reconstruction and original profile curves

(a) Reconstruction and original profile curves (b) Error between reconstruction and original profile curves

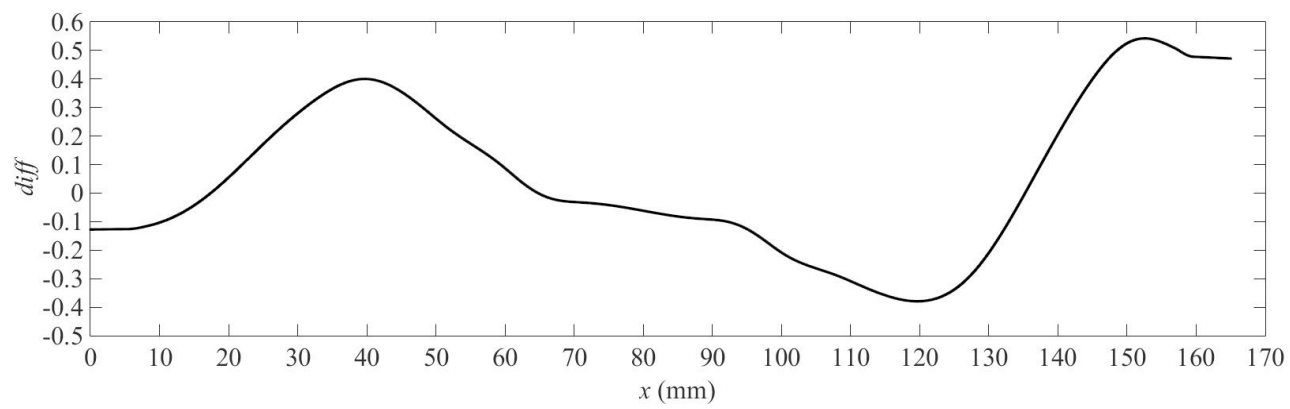

Fig. 8. First-order numerical differentiation of the reconstruction profile curve

\section{Conclusions}

To improve the repair quality and efficiency of a smooth part surface and disclose the relationship between a smooth profile curve measurement and reconstruction, this study analyzed the adaptive measurement, repair, smoothing, and curve reconstruction of smooth rotary parts on the basis of LOWESS and Akima interpolation theories. The following conclusions could be drawn:

(1) The measurement strategy based on the laser displacement sensor can adaptively measure the profile curve. However, the measurement data cannot be used directly for curve reconstruction and repair.

(2) The staged repair and smoothing strategy based on the LOWESS can effectively repair and smooth the abnormal data caused by the measurement error and profile defects, reduce the error between the repaired and the original curves, and decrease the difficulties in following up profile reconstruction.

(3) The profile curve can be reconstructed through Akima interpolation, thus obtaining a smooth repaired curve. In addition, the maximum error between the measurement and the original curves is not increased.

This study combines experiment and theoretical analysis and proposed a profile curve repair, smoothing, and reconstruction method using a laser displacement sensor. The construction platform is efficient and reliable. It provides an important guidance for improving part repair efficiency and accuracy and enhancing the automation level of part repair. In this study, the relationship between the measurement step length and the repair and smoothing 
effects is not analyzed in detail. The optimal selection of the measurement step length can be studied by designing a fullfactor experiment in the future.

\section{Acknowledgements}

This work was supported by the Education Scientific Research Project for the Young Teachers of Fujian Province, China (Grant No. JAT170613) and the Natural Science
Foundation for Fujian Institutions, China (Grant No. 2018J0176).

This is an Open Access article distributed under the terms of the Creative Commons Attribution License

\section{References}

1. Várady, T., Martin, R. R., Cox, J., "Reverse engineering of geometric models-an introduction". Computer-Aided Design, 29(4), 1997, pp.255-268.

2. Anwer, N., Mathieu, L., "From reverse engineering to shape engineering in mechanical design". CIRP Annals-Manufacturing Technology, 65(1), 2016, pp.165-168.

3. Raibulet, C., Fontana, F. A., Zanoni, M., "Model-Driven Reverse Engineering Approaches: A Systematic Literature Review". IEEE Access, 5, 2017, pp.14516-14542.

4. Gauthier, S., Puech, W., Bénière, R., Subsol, G., "Analysis of digitized 3D mesh curvature histograms for reverse engineering". Computers in Industry, 92-93,2017, pp.67-83.

5. Wang, Y., Xie, F., Ma, S., Dong, L., "Review of surface profile measurement techniques based on optical interferometry". Optics and Lasers in Engineering, 93, 2017, pp.164-170.

6. Wang, Z., "A one-shot-projection method for measurement of specular surfaces”. Optics Express, 23(3), 2015, pp.1912-1929.

7. Pan, B., Qian, K., Xie, H., Asundi, A., "TOPICAL REVIEW: Twodimensional digital image correlation for in-plane displacement and strain measurement: a review". Measurement Science \& Technology, 20(6), 2009, pp.152-154.

8. Murienne, B. J., Nguyen, T. D., "A comparison of 2D and 3D digital image correlation for a membrane under inflation". Optics and Lasers in Engineering, 77, 2016, pp.92-99.

9. Li, Q., Liu, Y., Niu, K., Liu, J., Chou, X., "Reconstruction of the 3D Surface Profile of Micro Device Based on White-Light Interference Measurement Technology". Journal of Test and Measurement Technology, 23(3), 2009, pp.201-204.

10. Angelo, L. D., Stefano, P. D., Pane, C., "Automatic dimensional characterisation of pottery". Journal of Cultural Heritage, 26, 2017, pp.118-128.

11. Tao, Y., Li, Y., Wang, Y. Q., Ma, Y. Y., "On-line point cloud data extraction algorithm for spatial scanning measurement of irregular surface in copying manufacture". International Journal of Advanced Manufacturing Technology, 87(5-8), 2016, pp.18911905.

12. Shinozaki, R., Sasaki, O., Suzuki, T., "Fast scanning method for one-dimensional surface profile measurement by detecting angular deflection of a laser beam". Applied Optics, 43(21), 2004, pp.41574163.

13. Paralı, L., Pechousek, J., Şabikoğlu, İ., Novak, P., Navarik, J., Vujtek, M., "A digital measurement system based on laser displacement sensor for piezoelectric ceramic discs vibration characterization". Optik-International Journal for Light and Electron Optics, 127(1), 2016, pp.84-89.

14. Giri, P., Kharkovsky, S., "Detection of Surface Crack in Concrete Using Measurement Technique With Laser Displacement Sensor". IEEE Transactions on Instrumentation \& Measurement, 65(8), 2016, pp.1951-1953.

15. Zheng, J., Li, Z., Chen, X., "Worn area modeling for automating the repair of turbine blades". International Journal of Advanced Manufacturing Technology, 29(10), 2006, pp.1062-1067.

16. Gao, J., Chen, X., Yilmaz, O., Gindy, N., “An integrated adaptive repair solution for complex aerospace components through geometry reconstruction". International Journal of Advanced Manufacturing Technology, 36(11-12), 2008, pp.1170-1179.

17. Bagci, E., "Reverse engineering applications for recovery of broken or worn parts and re-manufacturing: Three case studies". Advances in Engineering Software, 40(6), 2009, pp.407-418.
18. Yilmaz, O., Gindy, N., Gao, J., “A repair and overhaul methodology for aeroengine components". Robotics and Computer-Integrated Manufacturing, 26(2), 2010, pp.190-201.

19. Shen, C. H., Fu, H., Chen, K., Hu, S. M., "Structure recovery by part assembly". Acm Transactions on Graphics, 31(6), 2012, pp.111.

20. Jones, J., Mcnutt, P., Tosi, R., Perry, C., Wimpenny, D., "Remanufacture of turbine blades by laser cladding, machining and in-process scanning in a single machine". In: 23rd Annual International Solid Freeform Fabrication Symposium, Austin, USA: University of Texas, 2012, pp.821-827.

21. Wu, H., Gao, J., Li, S., Zhang, Y., Zheng, D., "A Review of Geometric Reconstruction Algorithm and Repairing Methodologies for Gas Turbine Components". TELKOMNIKA - Indonesian Journal of Electrical Engineering, 11(3), 2013, pp.1609-1618.

22. Wilson, J. M., Piya, C., Shin, Y. C., Zhao, F., Ramani, K., "Remanufacturing of turbine blades by laser direct deposition with its energy and environmental impact analysis". Journal of Cleaner Production, 80(80), 2014, pp.170-178.

23. Wu, Z., Zhong, Y., "Establishment of restoration model for damaged parts based on reverse engine". Machinery, 54(5), 2016, pp.41-43.

24. Huang, Y., Sun, W., Zhou, C., Huang, H., "Planning of repair area of visual damage on component to be remanufactured by laser cladding". Hanjie Xuebao/transactions of the China Welding Institution, 38(11), 2017, pp.51-56.

25. Li, L., Li, C., Tang, Y., Du, Y., "An integrated approach of reverse engineering aided remanufacturing process for worn components". Robotics and Computer-Integrated Manufacturing, 48, 2017, pp.39-50.

26. Sokół, K., Cekus, D., "Reverse Engineering as a Solution in Parts Restoration Process". Procedia Engineering, 177, 2017, pp.210217.

27. Zhang, X., Li, W., Cui, W., Liou, F., "Modeling of worn surface geometry for engine blade repair using Laser-aided Direct Metal Deposition process". Manufacturing Letters, 15, 2018, pp.1-4.

28. Zheng, Y., Qureshi, A. J., Ahmad, R., "Algorithm for remanufacturing of damaged parts with hybrid $3 \mathrm{D}$ printing and machining process". Manufacturing Letters, 15, 2018, pp.38-41.

29. Yi, Z., Pan, N., Liu, Y., Guo, Y., "Study of laser displacement measurement data abnormal correction algorithm". Engineering Computations, 34(1), 2017, pp.123-133.

30. Smolik, M., Skala, V., Nedved, O., "A Comparative Study of LOWESS and RBF Approximations for Visualization". In: Computational Science and Its Applications - ICCSA 2016, Beijing, China: Springer International Publishing, 2016, pp.405419.

31. Wang, Y., Yang, D., Liu, Y., "A real-time look-ahead interpolation algorithm based on Akima curve fitting". International Journal of Machine Tools and Manufacture, 85(5), 2014, pp.122-130.

32. Bica, A. M., "Optimizing at the end-points the Akima's interpolation method of smooth curve fitting". Computer Aided Geometric Design, 31(5), 2014, pp.245-257.

33. Luo, S. J., Fu, Y. T., Zhou, Y. X., "Perceptual matching of shape design style between wheel hub and car type". International Journal of Industrial Ergonomics, 42(1), 2012, pp.90-102. 